\title{
Características ergonômicas dos tratores agrícolas utilizados na região central do Rio Grande do Sul ${ }^{1}$
}

\author{
Ergonomic characteristics of agricultural tractors used in central region of \\ Rio Grande do Sul State, Brazil
}

\author{
Henrique Debiasi $^{2} \quad$ José Fernando Schlosser $^{3} \quad$ Eder Dornelles Pinheiro $^{4}$
}

\section{RESUMO}

A utilização de tratores agrícolas com boas condições ergonômicas reduz a probabilidade de ocorrência de acidentes e doenças ocupacionais, aumentando a eficiência dos trabalhadores. Neste trabalho, objetivou-se verificar a presença de itens relacionados à ergonomia de tratores agrícolas usados, bem como avaliar o comportamento destas características em função do tempo de uso das referidas máquinas. Foram analisados 175 tratores agrícolas pertencentes às microrregiões gaúchas de Santa Maria, Restinga Seca e Cachoeira do Sul, presentes em 114 propriedades aleatoriamente amostradas. Os resultados mostraram que apenas $3 \%$ dos espécimes avaliados eram equipados com cabina, sendo que a maior parte dos mesmos não apresentava isolamento do calor gerado pelo motor $e$ transmissão. Com relação à ergonomia dos comandos, mais de $70 \%$ dos tratores tinham alavancas de câmbio centrais, enquanto que apenas $8 \%$ eram equipados com volante de direção regulável. Da mesma forma, mais de $20 \%$ dos exemplares amostrados possuíam bancos sem estofamento. Além disso, a presença dos itens avaliados foi menor para os tratores mais antigos.

Palavras-chave: ergonomia, tratores agrícolas, normas técnicas, posto de operação.

\section{ABSTRACT}

It is possible to reduce the probability of work accidents, diseases occurrence and increase the work efficiency using agricultural tractors with good ergonomic conditions. The objective of this research was to verify the presence of ergonomic items in used agricultural tractors and also to evaluate the influence of tractor age in these characteristics. 175 agricultural tractors belonging to 3 regions of Rio Grande do Sul State (Santa Maria, Restinga Seca and Cachoeira do Sul, Brazil), present in 114 farms randomly sampled, were evaluated. The results showed that only 3\% of sampled specimens were equipped with a cab, and most part of the tractors did not have protection against the heat produced by tractor transmission and engine. With regard to ergonomics of commands, more than $70 \%$ of the tractors had gear levers positioned in its longitudinal central line, and only $8 \%$ were equipped with adjustable steering wheel. In the same way, more than $20 \%$ of sampled specimens did not have upholstered seat. Besides, the presence of the evaluated items were smaller for older tractors.

Key words: ergonomics, agricultural tractors, technical norms, workplace.

\section{INTRODUÇÃO}

A operação de tratores agrícolas é uma atividade que engloba basicamente dois fatores: o homem (operador) e a máquina (trator). Estes dois fatores interagem entre si, formando o sistema homem-máquina (MURREL, 1965; IIDA, 1990; GRANDJEAN, 1998). A eficiência com que o sistema homem-máquina executa suas funções depende de diversos fatores. A ergonomia age sobre estes fatores, buscando otimizá-los para aumentar a eficiência do sistema de forma a beneficiar o homem. MURREL (1965) expõe alguns destes fatores, entre os quais destacam-se, no âmbito da operação de tratores agrícolas, as condições ambientais do posto de operação (temperatura, luz, umidade do ar), ruídos, vibrações, comandos e assento do operador.

${ }^{1}$ Extraído da dissertação de mestrado do primeiro autor.

${ }^{2}$ Engenheiro Agrícola, MSc, Pesquisador, Centro de Pesquisa em Forrageiras, Fundação Estadual de Pesquisas Agropecuárias, BR-290, km 412, 97300-970, São Gabriel, RS. E-mail: henriquedebiasi@yahoo.com.br.

${ }^{3}$ Engenheiro Agricola, Doutor, Professor Titular, Departamento de Engenharia Rural, Centro de Ciências Rurais, Universidade Federal de Santa Maria, Campus Universitário Camobi, 97105-900, Santa Maria, RS. E-mail: schlosse@ccr.ufsm.br. Autor para correspondência.

${ }^{4}$ Acadêmico do Curso de Agronomia, Monitor da disciplina de Máquinas Agrícolas A. 
Quando a operação de tratores agrícolas não se constituir em um sistema homem-máquina eficiente, o operador é exposto a uma elevada carga física e mental. Isto resulta numa redução da eficiência do mesmo (produtividade e qualidade do trabalho), aumentando a ocorrência de erros, acidentes e o desenvolvimento de doenças ocupacionais (WITNEY, 1988; MÁRQUEZ, 1990; LILJEDAHL et al., 1996; YADAV \& TEWARI, 1998).

As características do assento do operador são de grande importância na tarefa de reduzir o trabalho estático muscular, oportunizando a tomada de posturas corporais corretas por parte do operador (MURREL, 1965; CUTULI et al. 1977). Trabalhos de pesquisa desenvolvidos por BOVENZI \& BETTA (1996), YADAV \& TEWARI (1998) e MEHTA \& TEWARI (2000) mostram que o trabalho estático gera fadiga muscular, o que aumenta o risco de ocorrência de acidentes de trabalho, além de potencializar a ocorrência de determinadas doenças ocupacionais no operador, como lombalgias e surgimento de hérnia de disco.

Entre as características que o assento do operador deve possuir, destacam-se as dimensões (largura e comprimento do assento, altura em relação à superfície de apoio, distância em relação ao volante de direção e os pedais e inclinação do assento e do encosto). Todas essas medidas encontram-se normalizadas através da norma NBR ISO 4253 (1999).

A análise da literatura disponível sobre ergonomia aplicada às máquinas agrícolas mostra que os comandos devem apresentar uma série de características dentro de determinados padrões, definidos por normas. Uma das principais é a de que posição dos comandos deve ser tal que permita um manejo fácil e seguro sem que seja necessário que o operador se desloque de sua posição normal de trabalho, ou seja, incline-se para algum lado (MÁRQUEZ, 1990). Dentre os comandos de um trator agrícola, o volante de direção merece atenção especial, por ser de acionamento contínuo. Além da distância em relação ao assento, outra característica importante deste comando é o grau de inclinação de seu eixo central em relação à vertical.

Assim, é importante que as condições ambientais no posto de operação dos tratores agrícolas sejam controladas. Neste sentido, já foram desenvolvidos sistemas que permitem isolar, pelo menos parcialmente, o operador do calor produzido pelo motor e pela transmissão, bem como daquele oriundo dos raios solares (toldo solar refletivo). Porém, o controle mais efetivo é, sem dúvida, aquele proporcionado por uma cabina. As cabinas colaboram ainda para a redução dos níveis de ruído, vibrações e substâncias estranhas presentes no ar (MÁRQUEZ, 1990; FEBO \& PESSINA, 1995; SCHLOSSER et al., 2001). A importância das cabinas é tal que, em alguns países (Inglaterra, Suécia e Finlândia), todos os tratores novos obrigatoriamente devem ser equipados de fábrica com cabinas que possuem sistema de calefação (SPRINGFELDT, 1996). Porém, na maioria dos países, grande parte dos tratores agrícolas ainda não possui cabina. Neste sentido, FEBO \& PESSINA (1995), em trabalho realizado na Itália, encontraram que apenas $24 \%$ dos tratores analisados estavam equipados com cabina. Porém, SCHLOSSER (2001) expõe que a agricultura brasileira, em geral, não tem condições de absorver o custo das cabinas, resultando numa baixa utilização deste dispositivo. Cabe ainda destacar que vários trabalhos de pesquisa mostram que, mesmo na Europa e nos Estados Unidos, a frota de tratores é considerada envelhecida (FEBO \& PESSINA, 1995; SPRINGFELDT, 1996; JANICAK, 2000). FMO (1974) expõe que máquinas antigas são mais perigosas que as mais modernas, devido ao desgaste natural das mesmas, o que aumenta a possibilidade de ocorrência de falhas mecânicas que podem culminar em acidentes, bem como ao fato de as máquinas mais modernas possuírem características ergonômicas e de segurança superiores às antigas.

O objetivo deste trabalho foi verificar a presença de alguns itens relacionados à ergonomia de tratores agrícolas usados, bem como avaliar o comportamento destas características em função do tempo de uso das referidas máquinas.

\section{MATERIAL E MÉTODOS}

A pesquisa foi executada em 21 municípios pertencentes a três microrregiões geográficas do Centro do Rio Grande do Sul: Santa Maria, Restinga Seca e Cachoeira do Sul representando $30 \%$ da área agrícola do estado e abrangia 114 empreendimentos agrícolas e aproximadamente 10.000 hectares. Para a determinação do tamanho da amostra, foi seguida a metodologia apresentada por STORCK et al. (2000), levando-se em consideração o coeficiente de variação (\%) obtido pelas diversas variáveis analisadas em levantamento piloto envolvendo 20 propriedades. O tamanho mínimo da amostra foi o maior entre os calculados para todas as variáveis determinadas, correspondendo a 153 tratores. O tipo de amostragem empregado, considerando as propriedades agrícolas visitadas, foi aleatória simples (SCHEAFFER et al., 1996), sendo que, dentro destas, o maior número possível de tratores agrícolas foi avaliado. 
O levantamento englobou 175 tratores agrícolas distribuídos em 12 marcas diferentes, entre as quais se encontram todas as que atualmente são comercializadas no mercado brasileiro.

A análise das condições ergonômicas se deu mediante a avaliação de itens referentes à ergonomia dos tratores agrícolas, como ano de fabricação, presença de toldo solar ou cabina, isolamento térmico do motor, isolamento térmico da transmissão, posicionamento das alavancas de câmbio, regulagem de inclinação do volante de direção,

Para a análise dos resultados, foram usados recursos de estatística descritiva, com apresentação tabular dos resultados. Para a comparação das freqüências absolutas das alternativas possíveis de cada variável, bem como para a análise de correlação com o tempo de uso das máquinas, foi utilizado o coeficiente de correlação simples.

\section{RESULTADOS E DISCUSSÃO}

Neste trabalho, definiu-se tempo de uso como sendo o número de anos que se passaram em relação ao ano de fabricação do trator. A análise estatística dos dados apresentados na tabela 1 indica que houve diferenças significativas entre as frequiências absolutas apresentadas por cada classe de tempo de uso, conforme o teste do quiquadrado a $1 \%$ de probabilidade de erro. Nota-se que a frota de tratores na região avaliada encontrase bastante envelhecida. SAAD (1983) considera 10 anos como sendo a vida útil normal dos tratores agrícolas. Conforme mostra a tabela 1 , cerca de $73 \%$ dos tratores amostrados foram fabricados há 11 ou mais anos. A média do tempo de uso dos tratores amostrados foi de 17,5 anos. O envelhecimento da frota de tratores na região onde foi executada esta pesquisa tem importância indiscutível, pois conforme FMO (1974) e FEBO \& PESSINA (1995), tratores muito antigos, além de terem sua produtividade comprometida e acarretarem elevados custos de manutenção, normalmente são caracterizados pela inexistência ou mau estado de conservação de itens referentes à ergonomia.

A proporção de tratores que apresentam toldo solar ou cabina, em função do tempo de uso dos mesmos, é dada igualmente pela tabela 1. Verifica-se que, quanto maior o tempo de uso, menor é a proporção dos tratores agrícolas amostrados que possuem toldo solar, enquanto que a proporção dos que não possuem nem toldo solar e nem cabina aumenta. A correlação entre a presença de toldo solar/ cabina e o tempo de uso foi significativa segundo o teste do qui-quadrado a $1 \%$ de probabilidade de erro.

A tabela 1 mostra ainda que as freqüências totais dos tratores que possuem toldo ou cabina ou não possuem nenhum dos dois dispositivos diferiram significativamente entre si, também segundo o teste do qui-quadrado a $1 \%$ de probabilidade de erro. Assim, a maior parte dos tratores agrícolas amostrados $(66,29 \%)$ estava equipada com toldo solar. Menos de $3 \%$ dos tratores envolvidos na pesquisa eram equipados com cabina. Outro dado interessante é que uma considerável proporção dos tratores amostrados (pouco mais de 27\%) não possui nenhum dos dois dispositivos. Neste caso, o operador encontra-se totalmente desprotegido, sem nenhum controle sobre as condições climáticas (poeira, sol, chuva, entre outros)

A colaboração do toldo solar, presente na maioria dos tratores analisados nesta pesquisa, limitase à proteção do operador do calor do sol e da chuva. Além disso, verificou-se, neste levantamento, que, dos 114 tratores equipados com toldo solar, $21(18,10 \%)$ este dispositivo não era original de fábrica e sim

Tabela 1 - Distribuição dos tratores avaliados quanto à presença de cabina, toldo, isolamento térmico do motor e da transmissão segundo a faixa de vida útil.

\begin{tabular}{|c|c|c|c|c|c|c|}
\hline \multirow[t]{2}{*}{ Anos de utilização } & \multirow[t]{2}{*}{ Freqüência \% } & \multicolumn{3}{|c|}{ Proteção do operador } & \multicolumn{2}{|c|}{ Isolamento térmico } \\
\hline & & Cabina & Toldo & Nenhum & Motor & Transmissão \\
\hline$<10$ & 26,86 & 8,51 & 91,49 & 0,00 & 80,85 & 70,21 \\
\hline 11 a 20 & 33,14 & 0,00 & 82,76 & 17,24 & 10,34 & 15,52 \\
\hline 21 a 30 & 32,00 & 1,79 & 39,29 & 58,93 & 1,79 & 1,79 \\
\hline 31 a 40 & 3,43 & 0,00 & 21,43 & 78,57 & 0,00 & 7,14 \\
\hline$>40$ & 4,57 & 0,00 & 0,00 & 0,00 & 0,00 & 0,00 \\
\hline$*$ & & $-0,73$ & $-0,98$ & 0,27 & $-0,78$ & $-0,80$ \\
\hline
\end{tabular}

* Coeficiente de Correlação entre a idade média dos tratores e a variável 
adaptado. Isso demonstra, em primeiro lugar, que muitos proprietários, especialmente os que também são operadores, sentem a necessidade de, pelo menos, se proteger da incidência direta dos raios solares. Em contrapartida, toldos solares não originais podem comprometer outros aspectos referentes à ergonomia e segurança, conforme explicam CUTULI et al. (1977).

Outro aspecto importante refere-se à incorporação de dispositivos que minimizem a exposição do operador ao calor gerado pelo próprio trator. Estes dispositivos correspondem ao isolamento térmico da transmissão e do motor. A tabela 1 mostra que a presença de isolamento térmico da transmissão e do motor tende a diminuir quanto mais antigo for o trator. Para ambos os itens, a correlação entre a presença e o tempo de uso foi significativa pelo teste do qui-quadrado a $1 \%$. Da mesma forma, a freqüência absoluta total dos tratores que apresentavam e não apresentavam isolamento térmico da transmissão e do motor diferiu significativamente entre si, conforme o mesmo teste estatístico. Assim, apenas 25,71\% e $24,82 \%$ dos tratores amostrados, respectivamente, eram equipados com dispositivos de isolamento térmico do motor e da transmissão.

Diante do que foi anteriormente exposto, verifica-se que os tratores em uso na região onde foi executada esta pesquisa, especialmente os mais antigos, não são capazes de oferecer um posto de operação com condições ambientais favoráveis ao operador. Este se encontra, na grande maioria dos casos, exposto em elevado grau a fatores adversos gerados pelo próprio trator e inerentes a fatores climáticos e elementos estranhos presentes no ar.

A tabela 2 apresenta a caracterização dos tratores agrícolas amostrados no que se refere ao posicionamento das alavancas de câmbio. Segundo esta tabela, a maior parte dos tratores agrícolas analisados $(69,71 \%)$ caracteriza-se por possuir alavancas de câmbio posicionadas no centro do posto de operação, entre as pernas do operador. Essas diferenças foram significativas pelo teste do quiquadrado a $1 \%$ de probabilidade de erro. Ainda conforme o mesmo teste estatístico, a proporção de tratores que possuem alavancas posicionadas lateralmente diminuiu significativamente para os tratores mais antigos.

Alavancas de câmbio posicionadas centralmente são desvantajosas comparativamente às posicionadas lateralmente. Estas, quando posicionadas no centro do posto de operação, tornam-se um obstáculo ao livre acesso e saída do operador, o que pode ocasionar acidentes.

Conforme a mesma tabela, a grande maioria dos tratores analisados ( $92 \%$ ) não é equipada com regulagem do volante de direção, diferindo significativamente da proporção de tratores que possuem este dispositivo, segundo o teste do quiquadrado a $1 \%$ de probabilidade de erro. Observa-se ainda que a presença de regulagem do volante de direção é praticamente nula nos tratores antigos, restringindo-se basicamente aos tratores com tempo de uso igual ou inferior a 10 anos. A ausência de regulagem do volante de direção é um problema ergonômico pronunciado. Além de ser o comando mais acionado, a inclinação de seu eixo vertical é de suma importância para o conforto do operador, sendo que, conforme especifica a NBR ISO 4253 (1999), não existe um valor ótimo determinado. A cada operador, deve ser dada a possibilidade de escolher esse parâmetro de acordo com suas características.

Os dados ainda especificados na tabela 2 mostram que uma proporção considerável dos tratores analisados não possui assento estofado (cerca de $21 \%$ ), embora a proporção dos que possuem esta característica tenha sido significativamente maior pelo

Tabela 2 - Distribuição dos tratores avaliados quanto à posição das alavancas, regulagem e estofamento do assento segundo a faixa de vida útil.

\begin{tabular}{llllll}
\hline \multirow{2}{*}{ Anos de utilização } & \multirow{2}{*}{ Freqüência $\%$} & \multicolumn{2}{c}{ Posição das alavancas } & Assento \\
\cline { 3 - 6 } & & Central & Lateral & Presença de regulagem & Presença de estofamento \\
\hline < 10 & 26,86 & 40,43 & 59,57 & 23,40 & 100,00 \\
11 a 20 & 33,14 & 75,86 & 24,14 & 1,72 & 87,93 \\
21 a 30 & 32,00 & 96,43 & 3,57 & 3,57 & 66,07 \\
31 a 40 & 3,43 & 78,57 & 21,43 & $0 *$ & 28,57 \\
$>40$ & 4,57 & n/a & n/a & n/a & n/a \\
$*$ & & 0,74 & $-0,74$ & $-0,81$ & $-0,97$ \\
\hline
\end{tabular}

n/a - Impossível avaliar este ítem

* Coeficiente de Correlação entre a idade média dos tratores e a variável 
teste do qui-quadrado a $1 \%$ de probabilidade de erro. Esta proporção foi bem superior aos $12,50 \%$ detectados por FEBO \& PESSINA (1995) em pesquisa realizada na Itália. Foi observado também que a ausência de estofamento no assento do operador se deu tanto por ser esta uma característica original quanto por ter sido depreciado e posteriormente retirado e não reposto pelo proprietário. Nota-se ainda que a proporção dos tratores agrícolas analisados cujo assento do operador não possui estofamento aumenta significativamente conforme aumenta o seu tempo de uso (teste do qui-quadrado a $1 \%$ ). Todos os tratores com 10 ou menos anos de uso apresentavam assento estofado, ao passo que, para os tratores com mais de 30 anos, mais de $70 \%$ não possuíam esta característica.

\section{CONCLUSÃo}

Os tratores agrícolas pertencentes às regiões estudadas estão, em sua maioria, excedendo a vida útil recomendada, sendo que aproximadamente $73 \%$ supera os dez anos de utilização;

a maior parte dos tratores agrícolas presentes na região não apresenta condições satisfatórias em termos de ergonomia, por não possuir elementos de conforto para o operador, como cabina e regulagem de volante;

a ampla maioria das características favoráveis de ergonomia tende a ser ausente nos tratores mais antigos.

\section{REFERÊNCIAS BIBLIOGRÁFICAS}

ASSOCIAÇÃO BRASILEIRA DE NORMAS TÉCNICAS. Tratores agrícolas - Acomodação do assento do operador Dimensões : NBR ISO 4253. Rio de Janeiro, 1999.

BOVENZI, M.; BETTA, A. Low-back disorders in agricultural tractor drivers exposed to whole-body vibration and postural stress. Journal of Safety Research, v.27, n.3, p.196-197, 1996.

CUTULI, J.A.; CAMPANUCCI, L.; TUSIANI, M.O. Seguridad y higiene en el trabajo. Buenos Aires : Instituto Argentino, 1977. $512 \mathrm{p}$
FEBO, P.; PESSINA, D. Survey of the working condition of used tractors in Northern Italy. Journal of Agricultural Engineering Research, v.62, p.193202, 1995.

FMO. Seguridad en la maquinaria agrícola. Moline : Deere \& Company, 1974. 326p.

GRANDJEAN, E. Manual de ergonomia. Trad.: João Pedro Stein. 4.ed. Porto Alegre : Artes Médicas, 1998.

IIDA, I. Ergonomia: projeto e produção. São Paulo : Edgard Blucher, 1990. 451p.

JANICAK, C.A. Occupational fatalities to workers age 65 and older involving tractors in the crops production agriculture industry. Journal of Safety Research, v.31, n.3, p.143-148, 2000

LILJEDAHL, J.B. et al. Tractors and their power units. 4.ed. St. Joseph : ASAE, 1996. p.203-232

MÁRQUEZ, L. Solo tractor’90. Madrid : Laboreo, 1990. 231p.

MEHTA, C.R.; TEWARI, V.K. Seating discomfort for tractor operators - a critical review. International Journal of Industrial Ergonomics, v.25, p.661-674, 2000

MURREL, K.F.H. Ergonomics: Man in his working environment. London : Chapman and Hall, 1965. 496p.

SAAD, O. Seleção do equipamento agrícola. 4.ed. São Paulo: Nobel, 1983. 126p.

SCHEAFFER, R.L.; MENDENHALL III, W.; OTT, R.L. Elementary survey sampling. 5.ed. Belmont : Duxbury, 1996. p.126-195.

SCHLOSSER, J.F. Tratores agrícolas. Santa Maria : UFSM, Departamento de Engenharia Rural, 2001. 63p. (Série técnica, I).

SPRINGFELDT, B. Rollover of tractors - international experiences. Safety Science, v.24, n.1, p. 95-110, 1996.

STORCK, L.; LOPES, S.J.; LÚCIO, A.D. Introdução à experimentação. Santa Maria: UFSM, 2000. 49p.

WITNEY, B. Choosing and using farm machines. Harlow : Longman Scientific and technical, 1988. p.28-94.

YADAV, R.; TEWARI, V.K. Tractor operator workplace design - a review. Journal of Terramechanics, v.35, p.4153, 1998. 